\title{
Physical Carboxymethylscleroglucan/Calcium Ion Hydrogels as Modified Drug Delivery Systems in Topical Formulations
}

\author{
Federica Corrente ${ }^{1}$, Pietro Matricardi ${ }^{1}$, Patrizia Paolicelli ${ }^{1}$, Beatrice Tita ${ }^{2}$, Federica Vitali ${ }^{2}$ \\ and Maria Antonietta Casadei ${ }^{1, *}$
}

1 Dipartimento di Chimica e Tecnologie del Farmaco, "Sapienza" Università di Roma, Piazzale Aldo Moro 5, 00185, Rome, Italy

2 Dipartimento di Fisiologia e Farmacologia, "Sapienza" Università di Roma, Piazzale Aldo Moro 5, 00185, Rome, Italy

* Author to whom correspondence should be addressed; E-mail: mariaantonietta.casadei@uniroma1.it

Received: 29 June 2009; in revised form: 16 July 2009/Accepted: 21 July 2009 /

Published: 24 July 2009

\begin{abstract}
A carboxymethyl derivative of scleroglucan (Scl-CM) with a $65 \pm 5 \%$ carboxylic group degree of derivatization (DD) was recently synthesized and characterized. Aqueous solutions of the polymer underwent to a sharp transition toward a gel like behaviour in the presence of divalent ions such as $\mathrm{Ca}^{+2}$. Physical hydrogels with different $\mathrm{Scl}-\mathrm{CM} / \mathrm{Ca}^{+2}$ ratios were prepared and characterized for their rheological behaviour. Their potential as drug delivery systems was also evaluated. To this end three non steroidal antiinflammatory drugs (NSAIDs) were loaded into the hydrogels obtained with $2 \% \mathrm{w} / \mathrm{v}$ solution of Scl-CM and 0.05 and $0.1 \mathrm{M} \mathrm{CaCl}_{2}$. The release rate of the drugs was critically related to the salt concentration. By an appropriate combination of the hydrogels prepared using different amounts of salt, it was possible to obtain a system able to release diclofenac with zero-order kinetics. Primary skin irritation tests showed a good biocompatibility of the new polymer, as well as of its hydrogels. These results suggest a potential of the new hydrogels for the development of modified delivery systems in topical formulations.
\end{abstract}

Keywords: carboxymethyl scleroglucan; physical hydrogels; controlled release; rheology; mucoadhesiveness; primary skin irritation 


\section{Introduction}

Polysaccharides and their derivatives represent a class of polymers widely used in pharmaceutical formulations. In particular they find employment as starting materials for the preparation of hydrogels, three-dimensional networks able to retain large amounts of water or biological fluids. As a consequence, these systems show a consistence very similar to natural living tissues that allows their use for several biomedical applications [1-4]. Among polysaccharides, scleroglucan (Scl) has been proposed for the preparation of effective modified drug delivery systems, a topic that has recently been reviewed [5]. Scl is a water-soluble extra-cellular polysaccharide produced by fungi of the genus Sclerotium. It consists of a backbone of $(1 \rightarrow 3)$ - $\beta$-linked glucose residues substituted with single $(1 \rightarrow 6)-\beta$-D-glucopyranosyl residues on every third backbone unit. Scl adopts in water a triple helix conformation that can be destroyed by dimethylsulfoxide and in solutions at $\mathrm{pH} \geq 12.5$ [6,7]. The controlled oxidation of Scl produces the opening of the glucose in the side chain with the formation of the aldehyde derivative [8] that can be further oxidized to the corresponding carboxylic acid (Sclerox) [9]. Sclerox is able to form chemical gels by reaction with 1,6-dibromohexane [10] or physical gels in the presence of calcium ions [5], both proposed as drug delivery systems.

Recently our group synthesized a carboxymethyl derivative (Scl-CM) of scleroglucan able to form physical hydrogels with divalent ions, such as $\mathrm{Ca}^{+2}$ [11]. Systems with different polymer/salt ratio were prepared and evaluated as controlled delivery systems of drugs, using acyclovir as a model. The release rate of the drug critically depended on the concentration of the salt and the molar ratio carboxylated repetitive units of the polymer/ $/ \mathrm{Ca}^{2+}$.

Starting from these preliminary results, we wish now to report further studies performed on Scl$\mathrm{CM}$ and its hydrogels, aimed at investigating their potential as drug delivery systems. First of all we planned to verify if the new systems could be used for the development of topical formulations containing drugs of different nature. To this end, some non steroidal anti-inflammatory drugs (NSAIDs) were chosen because of the several and well-known adverse effects associated to their systemic use, especially towards the gastrointestinal tract [12]. In order to avoid these side effects and prolong their activity, NSAIDs are often formulated as modified delivery systems in topical preparations. Three among the most used NSAIDs (ibuprofen, ketoprofen and diclofenac) were loaded into the hydrogels obtained from $2 \% \mathrm{w} / \mathrm{v}$ aqueous solutions of Scl-CM and two different concentration of $\mathrm{CaCl}_{2}(0.05$ and $0.1 \mathrm{M})$, and the corresponding release profiles were investigated. Moreover the possibility of developing a system able to release a drug according to zero-order kinetics was also evaluated. To this end diclofenac was selected, as it is available on the market in several topical preparations, such as gels, creams and patches. The rheological behaviour of the hydrogels containing or not diclofenac was investigated. Finally primary skin irritation studies were performed on rabbits.

\section{Results and Discussion}

\subsection{Synthesis of Scl-CM}

Scleroglucan is a neutral homopolysaccharide that can be modified by reaction with choloroacetic acid in basic media as previously reported $[11,13]$. In this way the neutral polysaccharide can be 
converted into a negatively charged polymer (Scl-CM). The synthesis scheme is shown in Figure 1.

Figure 1. Scheme of Scl-CM synthesis.

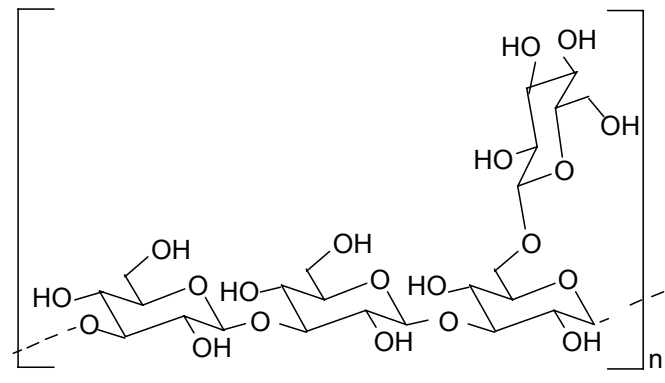

$\mathrm{Scl}$

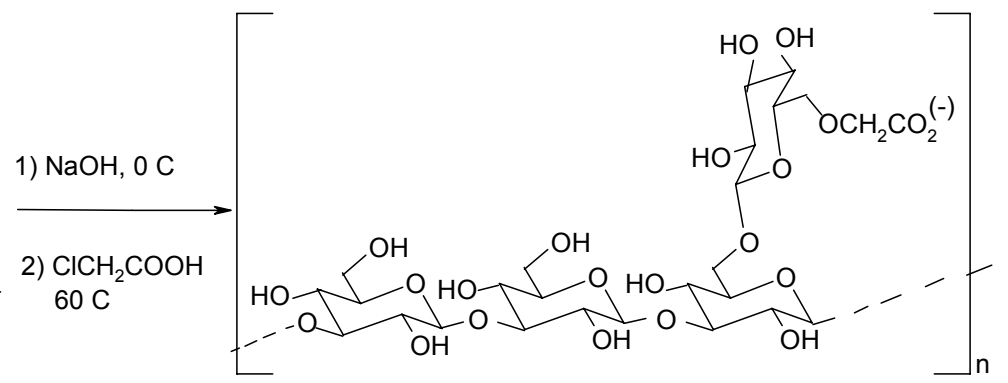

Scl-CM

The obtained polymer was characterized by FT-IR analysis. The spectra of the starting scleroglucan and of Scl-CM before and after the treatment on an ion-exchange resin are shown in Figure 2.

Figure 2. FT-IR spectra of Scl (A), Scl-CM before (B) and after (C) acidification.

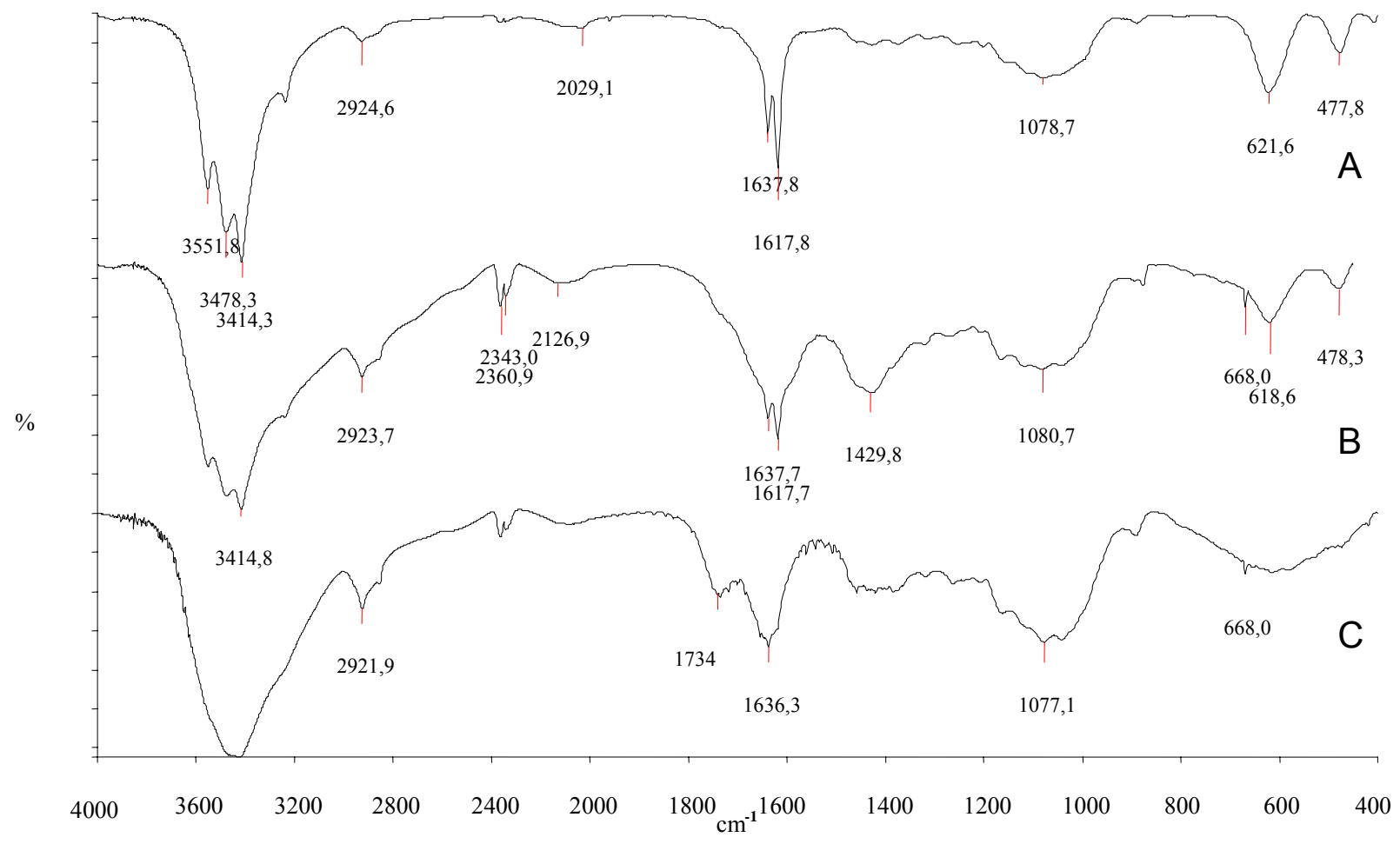

In the Scl-CM sample (B) a more intense band than in the Scl spectrum (A) can be observed at $1,618 \mathrm{~cm}^{-1}$, probably related to the asymmetric stretching vibration of the carboxylate group. The 
acidification of the derivative produces the disappearance of this peak and the appearance of a new peak at $1,734 \mathrm{~cm}^{-1}$, probably due to the stretching vibration of the $\mathrm{C}=\mathrm{O}$ in the undissociated acid.

\subsection{In vitro evaluation of the mucoadhesive properties of Scl-CM}

Mucoadhesion is a topic of current interest in the design of drug delivery systems. Mucoadhesive drug delivery systems prolong the residence time of the dosage form at the site of application and facilitate an intimate contact of the dosage form with the underlying absorption surface, thus contributing to an improved therapeutic performance of the drug. Therefore, the mucoadhesive properties of the new polymer were investigated. The ability of Scl and Scl-CM to interact with mucin was evaluated by a turbidimetric method and compared with hyaluronic acid, used as a positive control. Since Scl and Scl-CM do not produce clear solutions, neither in distilled water nor in phosphate buffer, the polymers were centrifuged before the assays, in order to remove eventual macro-aggregates that would interfere with the turbidimetric evaluation. Then the polymers were added to a $1 \% \mathrm{w} / \mathrm{v}$ mucin suspension and after $30 \mathrm{~min}, 2,4,6,8$ and 24 hours of incubation, the transmittance values of the polymer-mucin mixtures were determined at $500 \mathrm{~nm}$. The results are reported in Figure 3.

Figure 3. Transmittance \% values at $500 \mathrm{~nm}$ of mucin dispersions in the presence of Scl $(\diamond)$, Scl-CM ( $\bullet$ ) and hyaluronic acid, HA $(\boldsymbol{\Delta})$ chosen as a positive control, as a function of the time.

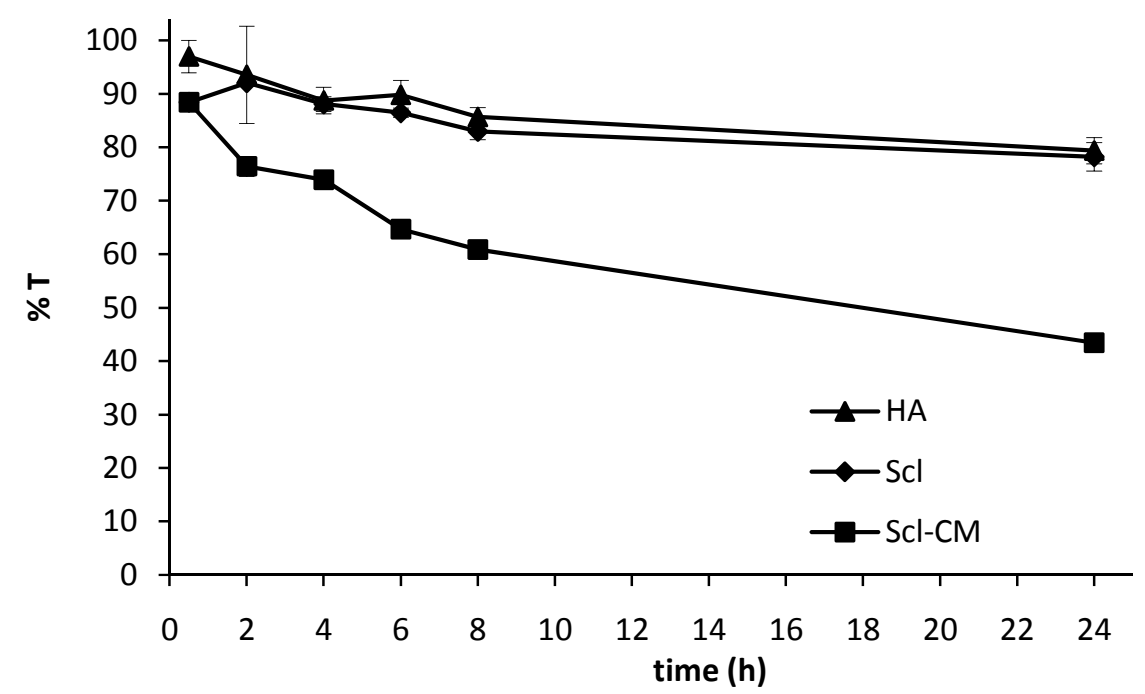

The greater the interaction between the polymer and mucin is, the lower is the transmittance value of the tested sample (greater UV-Vis scattering), due to the formation of macro-aggregates [14,15]. Therefore it can be observed that under the adopted experimental conditions, Scl presents the same ability to interact with mucin as hyaluronic acid, whereas the insertion of carboxylic groups on its backbone caused a marked increase in the mucoadhesive properties of the polymer. In fact, lower transmittance values were measured for the Scl-CM/mucin mixture than for Scl and $\mathrm{HA} /$ mucin ones, probably because the presence of the carboxylic groups increases the hydrogen bonds responsible for the polymer-mucin interactions. 


\subsection{Release studies from physical hydrogels of Scl-CM}

Physical hydrogels of Scl-CM were prepared taking into account a previous report [11], where preliminary studies were performed in order to find the experimental conditions for the hydrogels formation. In that work two polymer concentrations ( 1 and $2 \% \mathrm{w} / \mathrm{v})$ and different amounts of salt were investigated. On the basis of these preliminary studies, the $2 \% \mathrm{w} / \mathrm{v}$ concentration was now chosen for further investigation, because it allowed us to explore a wider range of $\mathrm{CaCl}_{2}$ concentrations. In particular hydrogels with $0.05,0.10,0.25$ and $0.50 \mathrm{M}$ of $\mathrm{CaCl}_{2}$ and loaded with $0.25 \% \mathrm{w} / \mathrm{v}$ acyclovir can be prepared in these conditions. The release rate of the drug from the four Scl-CM hydrogels $\left(\mathrm{C}_{\mathrm{p}} 2-\right.$ $\left.\mathrm{C}_{\mathrm{s}} 0.50 ; \mathrm{C}_{\mathrm{p}} 2-\mathrm{C}_{\mathrm{s}} 0.25 ; \mathrm{C}_{\mathrm{p}} 2-\mathrm{C}_{\mathrm{s}} 0.10 ; \mathrm{C}_{\mathrm{p}} 2-\mathrm{C}_{\mathrm{s}} 0.05\right)$ was studied in phosphate buffer $(\mathrm{PB})$ at $\mathrm{pH}=7.4$ and the results reported in Table 1.

Table 1. Percentages of acyclovir released from Scl-CM based hydrogels with different salt concentration in 6 and 24 hours $\left(\mathrm{C}_{\mathrm{p}}=\right.$ polymer concentration; $\mathrm{C}_{\mathrm{s}}=$ salt concentration). The results were reported as mean value $\pm \mathrm{SD}$.

\begin{tabular}{ccc}
\hline Sample & $\begin{array}{c}\text { Drug released in } \mathbf{6 ~ h} \\
\mathbf{( \% )}\end{array}$ & $\begin{array}{c}\text { Drug released in } \mathbf{2 4} \mathbf{~ h} \\
\mathbf{( \% )}\end{array}$ \\
\hline $\mathrm{C}_{\mathrm{p}} 2-\mathrm{C}_{\mathrm{s}} 0.50$ & $16.2 \pm 1.9$ & $21.5 \pm 1.2$ \\
$\mathrm{C}_{\mathrm{p}} 2-\mathrm{C}_{\mathrm{s}} 0.25$ & $40.2 \pm 2.5$ & $63.6 \pm 0.5$ \\
$\mathrm{C}_{\mathrm{p}} 2-\mathrm{C}_{\mathrm{s}} 0.10$ & $90.6 \pm 1.2$ & $98.5 \pm 1.2$ \\
$\mathrm{C}_{\mathrm{p}} 2-\mathrm{C}_{\mathrm{s}} 0.05$ & $92.7 \pm 0.5$ & $99.0 \pm 0.3$ \\
\hline
\end{tabular}

Furthermore for an easier comparison, the release profiles of acyclovir from the four systems are reported in Figure 4.

Figure 4. Release profiles $\left[\left(\mathrm{M}_{\mathrm{t}} / \mathrm{M}_{\infty}\right) \times 100\right]$ of acyclovir from hydrogels $\mathrm{Cp} 2-\mathrm{Cs} 0.05(\mathbf{\Delta})$, $\mathrm{Cp} 2-\mathrm{Cs} 0.10(\bullet), \mathrm{Cp} 2-\mathrm{Cs} 0.25(\bullet)$ and $\mathrm{Cp} 2-\mathrm{Cs} 0.50(\bullet)$, maintained in PB solution ( $\mathrm{pH}=7.4)$ at $37.0 \pm 0.1^{\circ} \mathrm{C}$ for 6 hours.

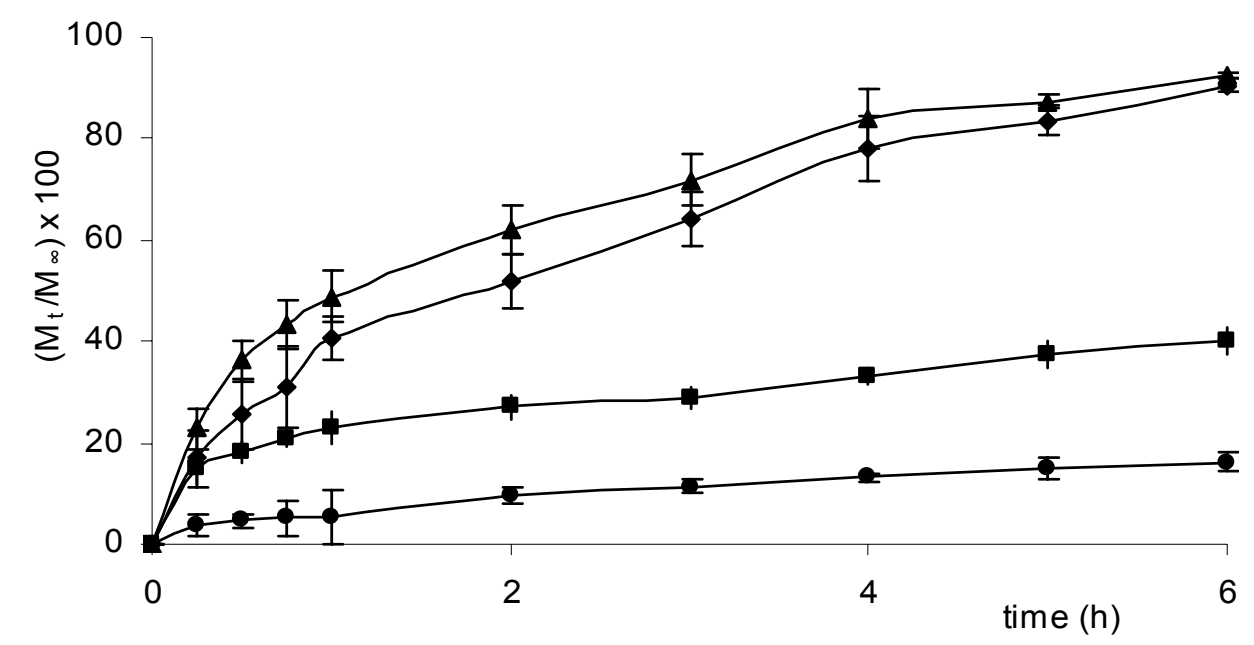


As it can be observed, the release rate of acyclovir depends critically on the amount of salt used to prepare the matrices - the higher the $\mathrm{CaCl}_{2}$ concentration, the slower the release. The matrices having the higher salt concentrations were unable to release sufficient amounts of drug either in $6 \mathrm{~h}$, the usual permanence time of topical formulations, nor in $24 \mathrm{~h}$, the maximum application time of transdermal patches. On the contrary, a quite complete release was observed from hydrogels prepared using salt concentrations lower than $0.1 \mathrm{M}$. As a consequence, systems with 0.05 and $0.1 \mathrm{M} \mathrm{CaCl}_{2}$ were considered for further investigation because they allowed the quantitative release of the drug in $24 \mathrm{~h}$.

Figure 5. Release profiles $\left[\left(\mathrm{M}_{\mathrm{t}} / \mathrm{M}_{\infty}\right) \times 100\right]$ of ketoprofen from hydrogels Cp2-Cs0.05 (») and Cp2-Cs0.10 ( $\square$ ) maintained in PB solution $(\mathrm{pH}=7.4)$ at $37.0 \pm 0.1{ }^{\circ} \mathrm{C}$ for 24 hours.

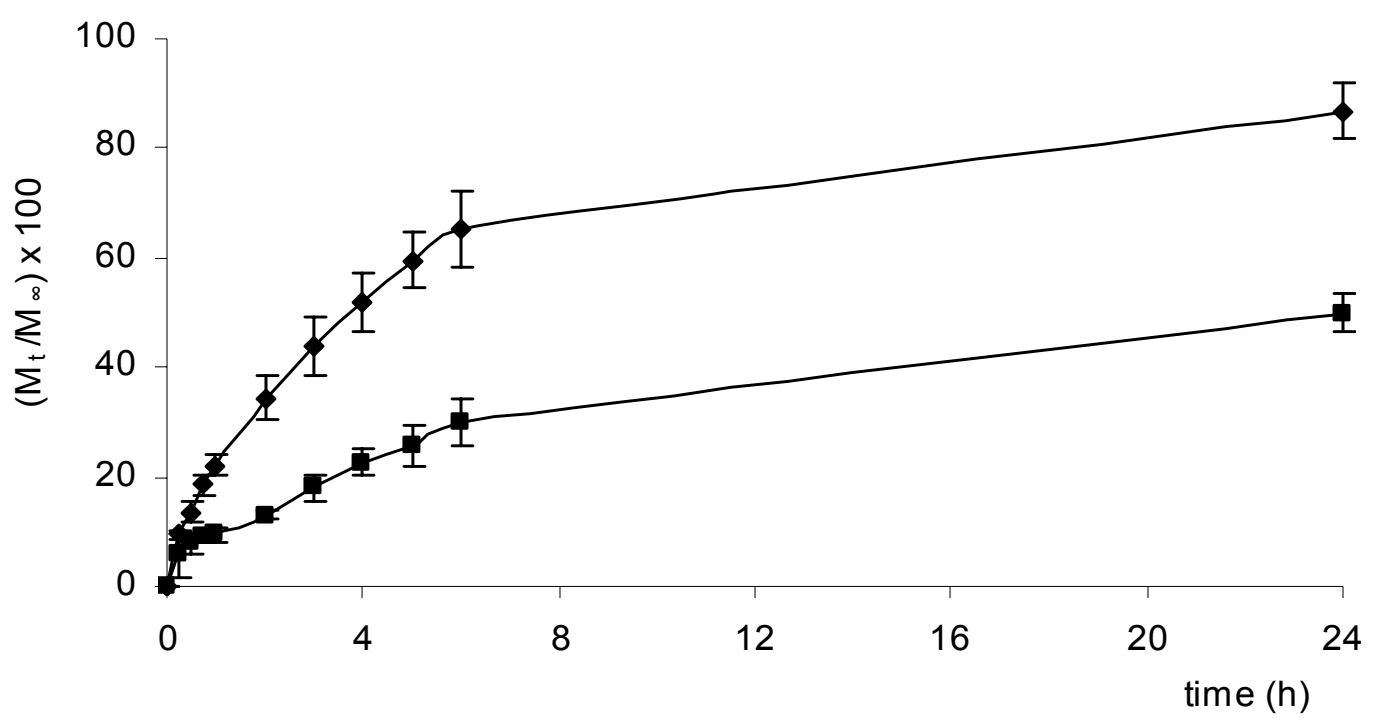

Figure 6. Release profiles $\left[\left(\mathrm{M}_{\mathrm{t}} / \mathrm{M}_{\infty}\right) \times 100\right]$ of ibuprofen from hydrogels $\mathrm{Cp} 2-\mathrm{Cs} 0.05(\boldsymbol{\Delta})$ and $\mathrm{Cp} 2-\mathrm{Cs} 0.10(\diamond)$ maintained in $\mathrm{PB}$ solution $(\mathrm{pH}=7.4)$ at $37.0 \pm 0.1^{\circ} \mathrm{C}$ for 24 hours.

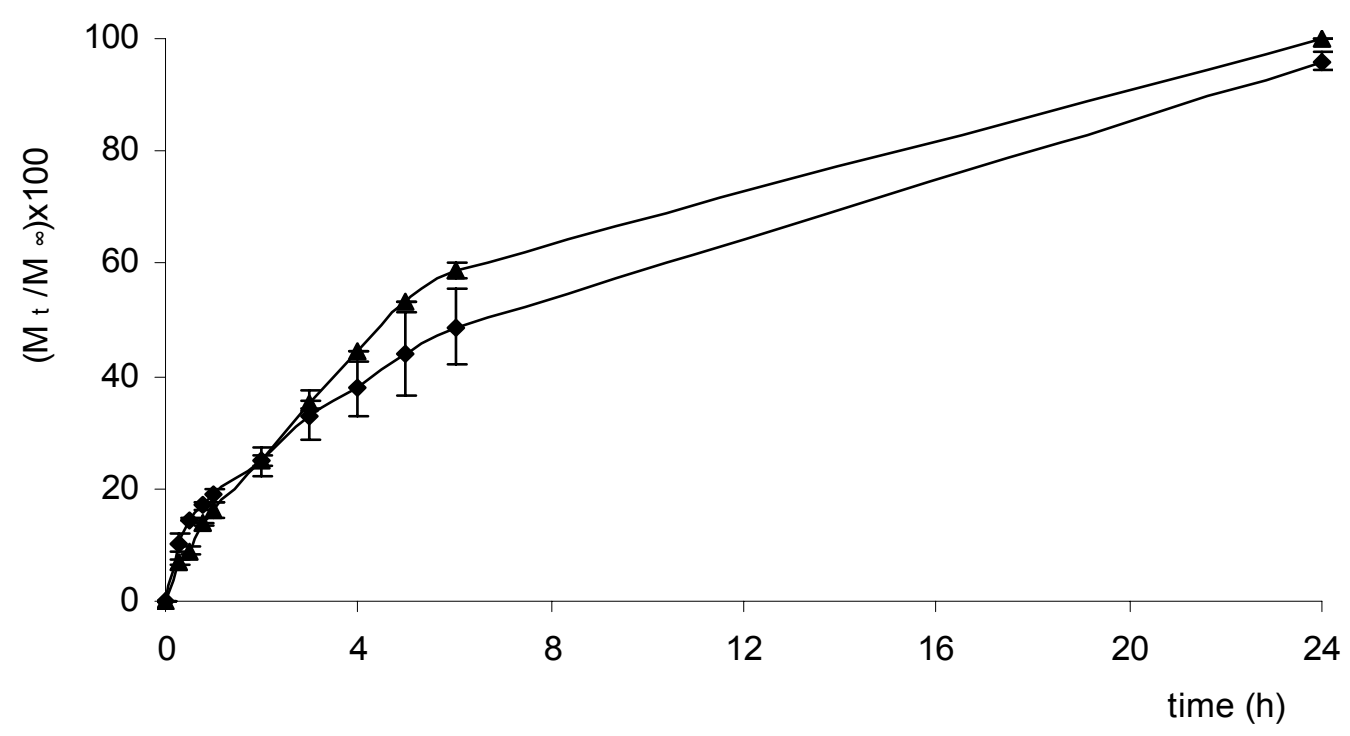


Figure 7. Release profiles $\left[\left(\mathrm{M}_{\mathrm{t}} / \mathrm{M}_{\infty}\right) \times 100\right]$ of diclofenac from hydrogels Cp2-Cs0.05 ( $\left.\boldsymbol{\Delta}\right)$ and $\mathrm{Cp} 2-\mathrm{Cs} 0.10(\mathbf{m})$ maintained in PB solution $(\mathrm{pH}=7.4)$ at $37.0 \pm 0.1^{\circ} \mathrm{C}$ for 24 hours.

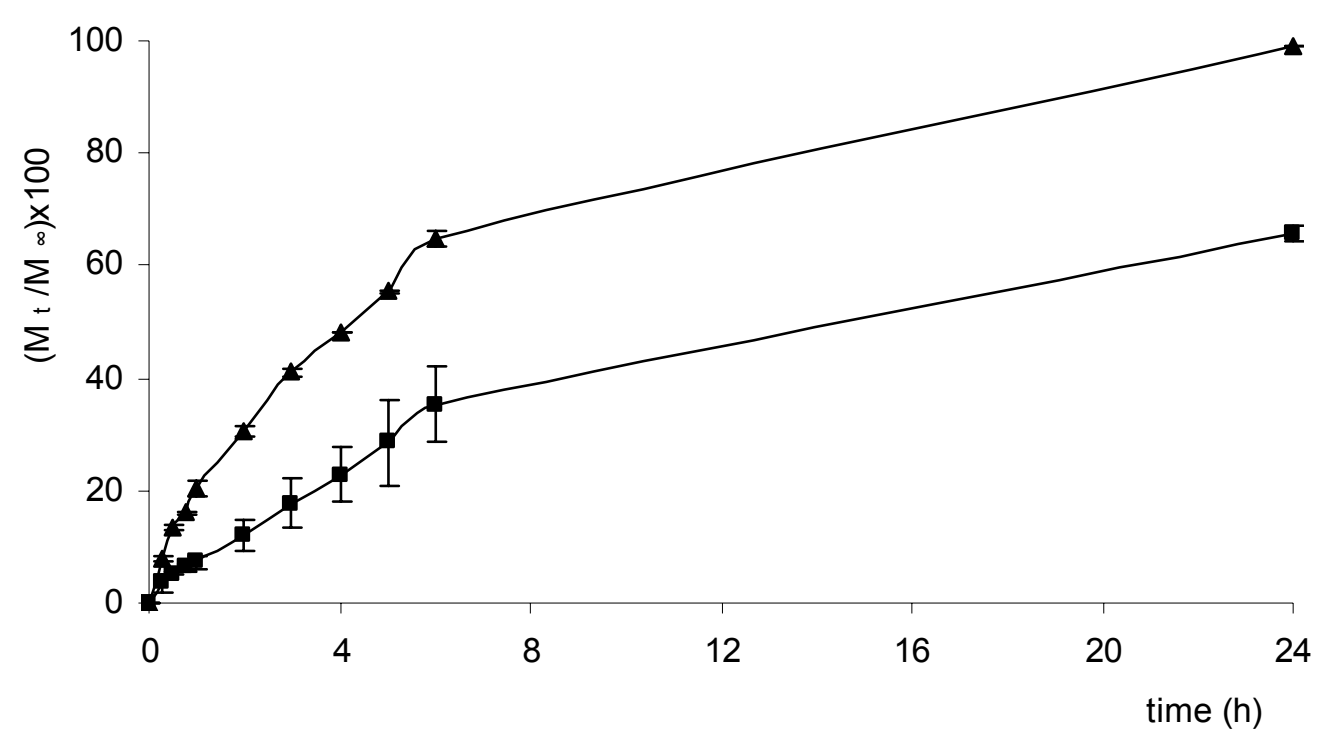

Three anti-inflammatory drugs (NSAIDs) - ketoprofen, ibuprofen and diclofenac - among the most widely used for oral as well as for topical administration, were loaded into the two selected matrices (Cp2-Cs0.1 and Cp2-Cs0.05). As stated before, NSAIDs are often formulated for topical applications, for example in gels and patches, in order to avoid their well-known adverse effects associated with their systemic use, especially towards the gastrointestinal tract. In fact a high incidence of gastric and duodenal ulcerations has been reported for their oral use [12]. The release profiles of ketoprofen, ibuprofen and diclofenac from Scl-CM based hydrogel are reported in Figures 5-7.

As already observed for acyclovir, even for these drugs the higher the salt concentration is, the slower is the release rate. Differences in the release rates of ibuprofen respect to ketoprofen and diclofenac may probably be related to differences in structure and dimensions of the molecules. Experiments performed in medium at $\mathrm{pH}=5.5$, miming skin conditions, showed a release rate only slightly slower than that obtained in PB solution, as already reported for acyclovir [11].

\subsection{Release studies of diclofenac from a double-layered system}

As observed before, the release rate of a drug from $\mathrm{Scl}-\mathrm{CM} / \mathrm{CaCl}_{2}$ hydrogels could be easily modified changing the salt concentration. It is well known that systems able to release a drug according to zero-order kinetics could be very useful for long-term medications, reducing the number of daily applications. Therefore, we investigated the possibility of developing a sustained delivery system based on $\mathrm{Scl}-\mathrm{CM} / \mathrm{CaCl}_{2}$ hydrogels by combining hydrogels at different salt concentration. To this aim a double-layered sample constituted by an outer layer of a looser hydrogel ( $\mathrm{Cp} 2-\mathrm{Cs} 0.05)$ and an inside layer of a stronger hydrogel $(\mathrm{Cp} 2-\mathrm{Cs} 0.10)$ both loaded with diclofenac sodium salt $(0.5 \%$ $\mathrm{w} / \mathrm{v}$ ), was prepared. The release profile of diclofenac from this system is reported in Figure 8 as the amount of the released drug as a function of the time. 
Figure 8. Release profile of diclofenac from the system at double-layer maintained in PB solution $(\mathrm{pH}=7.4)$ at $37.0 \pm 0.1^{\circ} \mathrm{C}$ for 24 hours.

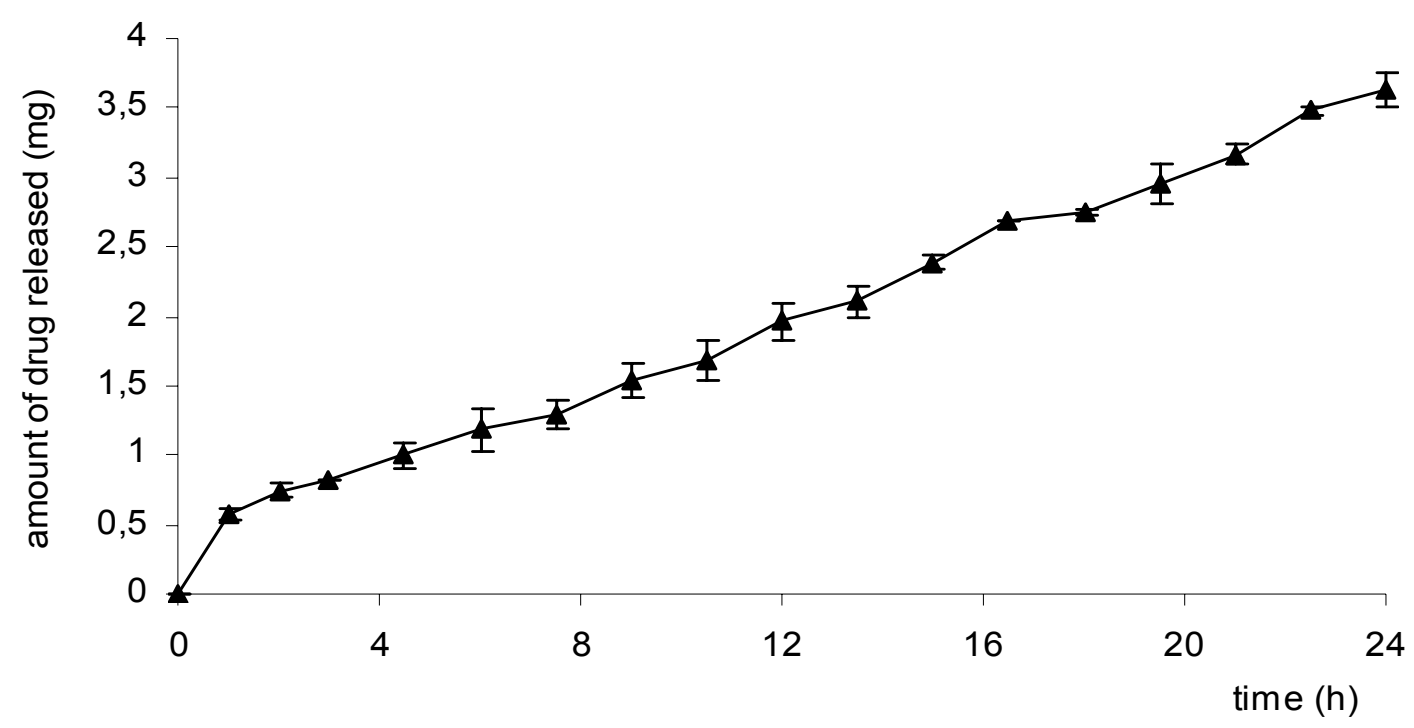

A burst effect is evident at the first hour, soon followed by a zero-order release kinetic. It is likely that when the outer layer becomes depleted in the drug, it is replaced by other drug coming from the deeper layer with a slower rate, so the system is able to release a constant amount of diclofenac for at least 24 hours after the application. As a consequence the combination of layers of hydrogels at different salt concentration could constitute a very easy method to build a system able to release the drug at the rate and in the amount requested by the therapeutic necessities.

\subsection{Rheological measurements}

The rheology of a topical formulation is among the most important properties to be measured, since it influences the ability to spread over the skin and mucosae and it can also affect the performance of the formulation. Therefore the rheological behaviour of Scl-CM based hydrogels was evaluated. To this end aliquots of $\mathrm{CaCl}_{2}$ water solutions were added to samples of $2 \% \mathrm{w} / \mathrm{v} \mathrm{Scl}-\mathrm{CM}$ water solutions in order to obtain hydrogels with salt concentrations of $0.05,0.1,0.25$ and $0.5 \mathrm{M}$; the thus obtained hydrogels were submitted to rheological investigation.

The rheological properties of the hydrogels obtained with $2 \% \mathrm{w} / \mathrm{v}$ Scl-CM were not influenced by the temperature, so all the experiments were performed at room temperature [16]. In Figure 9 the mechanical spectra, i.e. the elastic $\left(G^{\prime}\right)$ and the loss $\left(G^{\prime}\right.$ ') moduli profile as a function of the mechanical shear deformation applied to the samples at different frequencies, are reported for each hydrogel prepared. The quite parallel profile of the $G^{\prime}$ and $G$ ' curves for each concentration is a typical behaviour of a hydrogel. As shown in the Figure 9, an increase of the salt concentration results in an increase of the G' and the G'. In detail, the G' value, that is a measure of the strength of the gel, increases as the $\mathrm{CaCl}_{2}$ increases from $0.05 \mathrm{M}$ to $0.25 \mathrm{M}$. The sample $\mathrm{Cp} 2-\mathrm{Cs} 0.50$, i.e. with the highest concentration of salt, shows the G' value almost identical to Cp2-Cs0.25 one, evidencing that at 0.25 $\mathrm{M}$ the maximum interaction between polymer and salt is reached. 
Figure 9. Mechanical spectra of the hydrogels Cp2-Cs0.05 (G'», G’ $\diamond), \mathrm{Cp} 2-\mathrm{Cs} 0.10$ $\left.\mathrm{G}^{\prime}, \square \mathrm{G}, '\right), \mathrm{Cp} 2-\mathrm{Cs} 0.25\left(\bullet \mathrm{G}^{\prime}, \circ \mathrm{G}^{\prime \prime}\right)$ and $\mathrm{Cp} 2-\mathrm{Cs} 0.50\left(\boldsymbol{\Delta} \mathrm{G}^{\prime}, \Delta \mathrm{G}^{\prime \prime}\right)$ at $25.0 \pm 0.2{ }^{\circ} \mathrm{C}$.

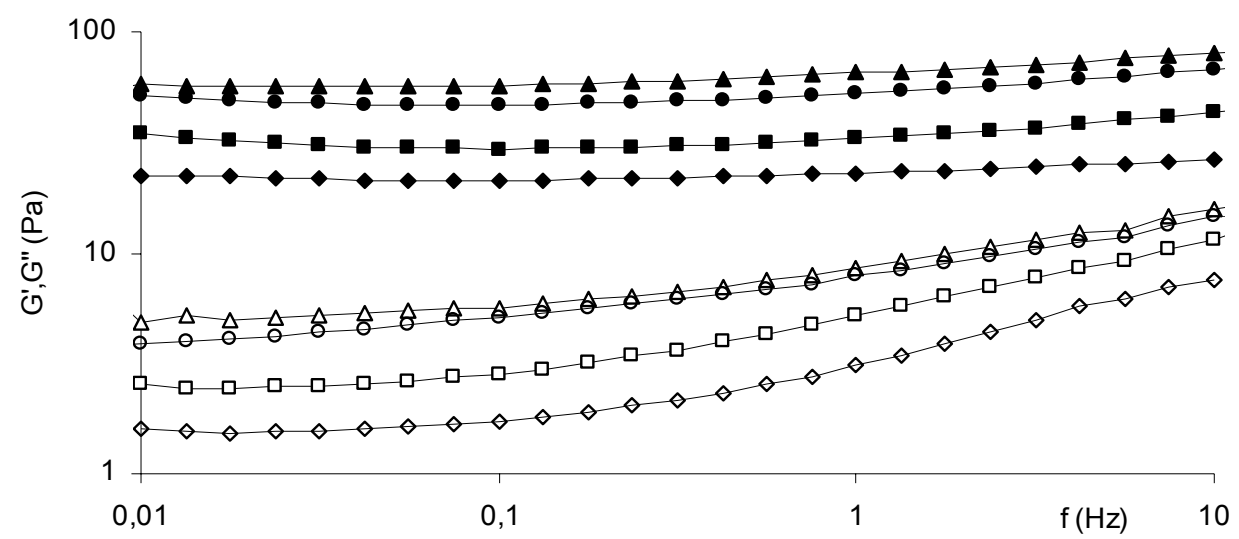

The viscosity $(\eta)$ as a function of the shear rate $\left(\gamma^{*}\right)$ for each hydrogel was also recorded in order to assess the ability of the systems prepared to be spread for topical applications. The physical hydrogels are able to flow and a pseudoplastic behaviour was observed. In Figure 10 (A) the flow curves of two of the hydrogels prepared $(\mathrm{Cp} 2-\mathrm{Cs} 0.05$ and $\mathrm{Cp} 2-\mathrm{Cs} 0.1)$ are reported. At very low shear rate the values of the shear viscosity are above $10^{3}$ Pa.s, whereas above $10 \mathrm{~s}^{-1}$, in the range of shear rates usually adopted during topical applications, the shear viscosity drops at values lower than $10^{1} \mathrm{~Pa}$.s.

In Figure 10 (B) the same experiments were carried out on hydrogel loaded with diclofenac. The behaviour appear to be quite similar; at low shear rate the shear viscosity of the hydrogel was affected by the presence of the drug with respect to the unloaded hydrogel.

Figure 10. Flow curves of the hydrogels unloaded (A) and loaded (B) with diclofenac sodium salt: $\square \mathrm{Cp} 2-\mathrm{Cs} 0.05$ and $\boldsymbol{\Delta} \mathrm{Cp} 2-\mathrm{Cs} 0.1$ at $25 \pm 0.2{ }^{\circ} \mathrm{C}$.

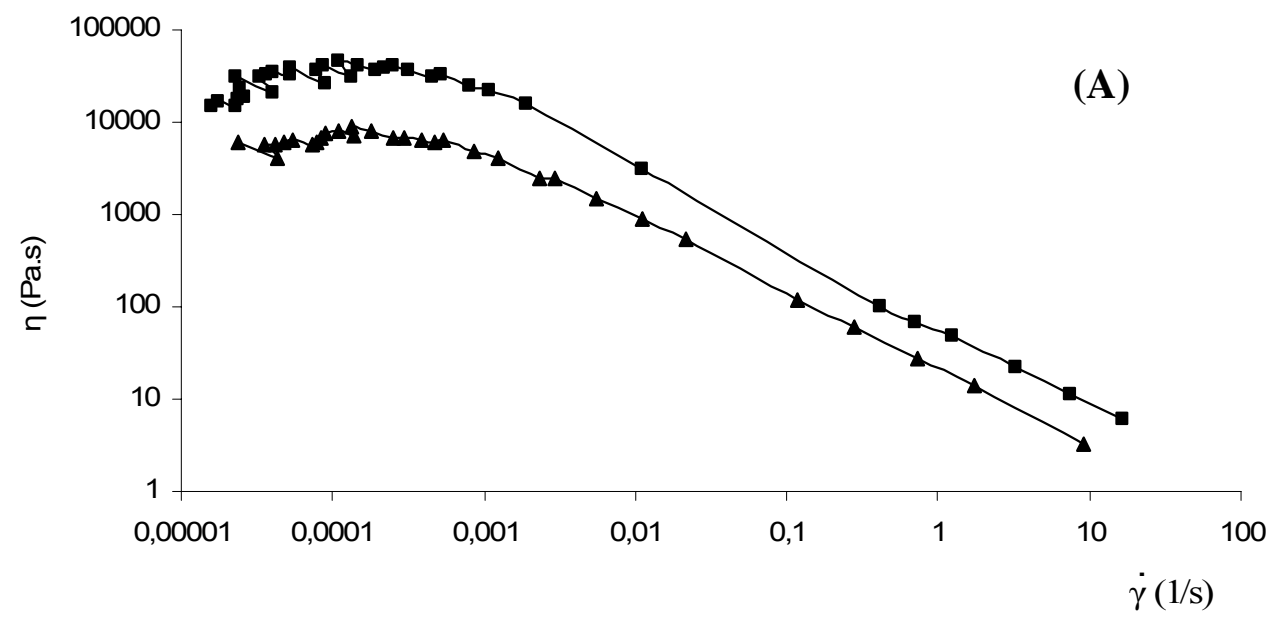


Figure 10. Cont.

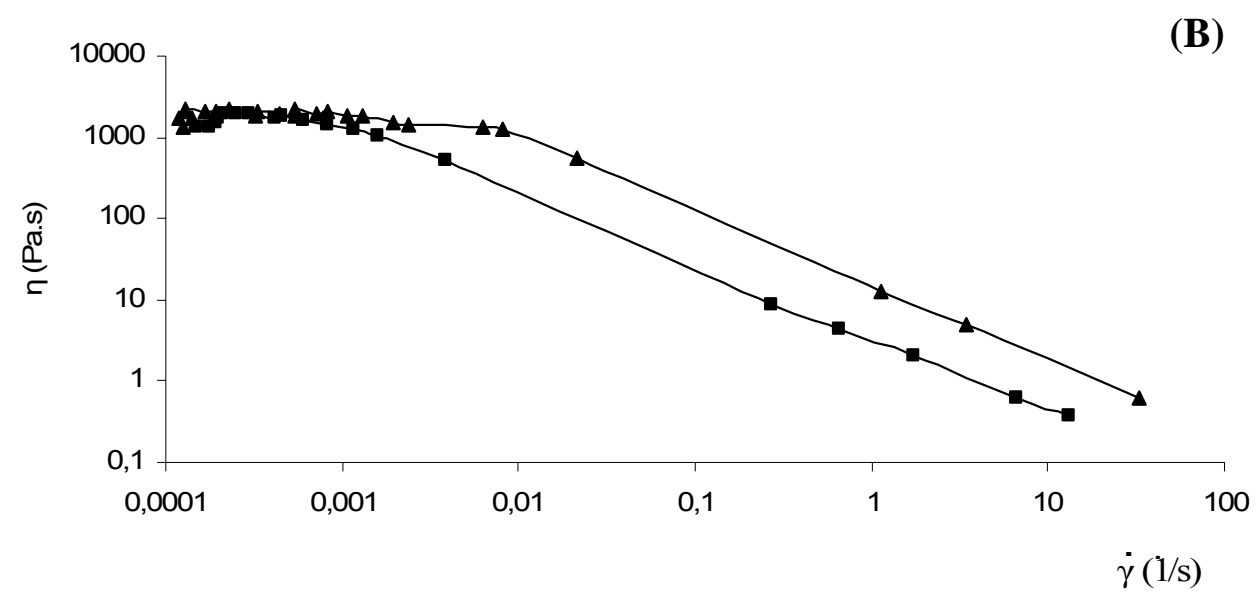

The effect of the drug loading on the shear rate viscosity of the hydrogels, with a reduction of the absolute values with respect to the unloaded hydrogels, can be ascribed to the disturbing effect on the network formation exerted by the host molecules. This negative interaction between the drug molecules and the network structure is more evident in the low shear rate regime region, because the measurements performed applying small deformations, preserve the overall loaded hydrogel structure. In this conditions the more structured hydrogel, i.e. the one with the higher salt concentration ( $G^{\prime}$ increases as the $\mathrm{CaCl}_{2}$ concentration is increased, see Figure 9) experiences deeper modification. From a physical point of view, this behaviour could be related to a limitation of the self-structuring ability of the $\mathrm{Scl}-\mathrm{CM} / \mathrm{CaCl}_{2}$ system due to the host molecules, thus levelling the low shear viscosity among the systems.

On the other hand, at higher shear rate conditions, the networks are in a large deformations regime; in this case, in the framework above depicted, the networks are continuously modified and the onset of the pseudoplasticity is more evident for the system with the higher $\mathrm{CaCl}_{2}$ concentration because of a reduced mobility of the Scl-CM chains. The overall results suggest that the spread-ability and the adherence of the materials is suitable for the application and performance on the skin and mucosal surfaces [17].

\subsection{Primary skin irritation experiments}

Primary skin irritation experiments were carried out in order to verify a possible use of the new polymer and of its physical hydrogels in topical formulations. The tests were performed on rabbits. Individual skin irritation score results, calculated as described in the Experimental part, were always equal to 0 . In the animals erythema or edema were absent after 24,48 and $72 \mathrm{~h}$ of application of the three samples ( $\mathrm{Scl}, \mathrm{Scl}-\mathrm{CM}$ and $\mathrm{Scl}-\mathrm{CM} / \mathrm{Ca}^{2+}$ ) as well as in the control (Figure 11). According to our data, the Primary Skin Irritation Index (PII) of the tested materials was negligible, showing the absence of skin irritation, so that they could be useful as modified drug delivery system in topical formulations. 
Figure 11. The back of the animals clipped and divided into four sites, after $24 \mathrm{~h}$ from the application of Scl, Scl-CM and Scl-CM/CaCl 2 .

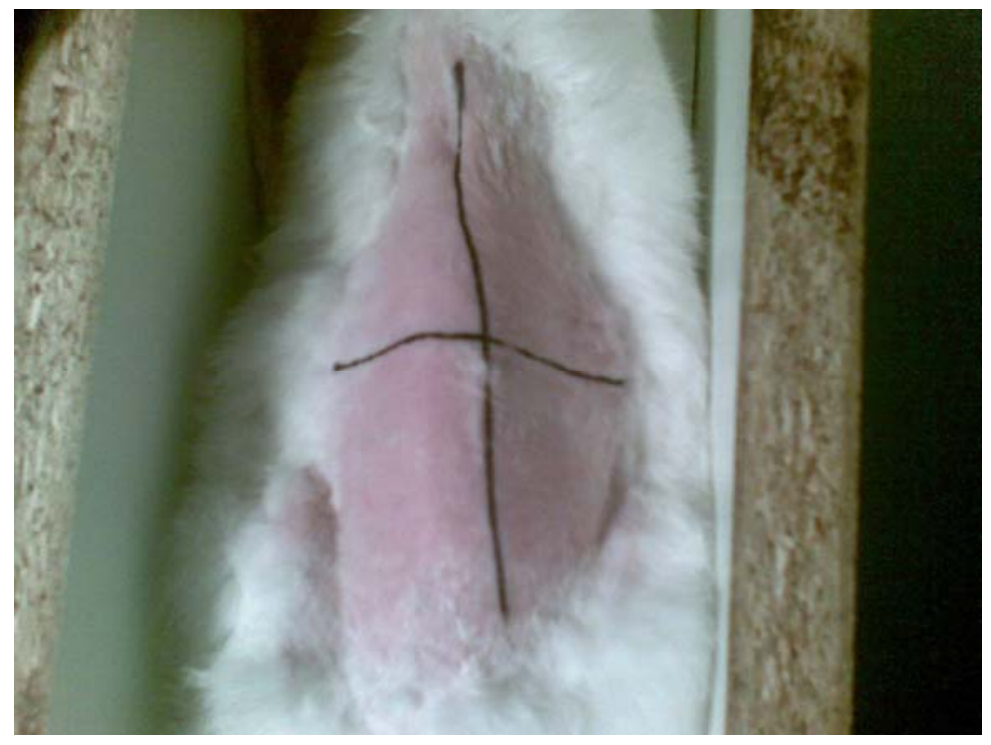

\section{Experimental Section}

\subsection{Materials}

All used reagents were of analytical grade. Scleroglucan with $\mathrm{Mw}=1.4 \times 10^{6}$ as evaluated by viscosimetric measurements in $0.01 \mathrm{M} \mathrm{NaOH}$, was provided by Carbomer. Dimethylsulfoxide (DMSO), $\mathrm{CaCl}_{2} \times 2 \mathrm{H}_{2} \mathrm{O}$, ibuprofen sodium salt and chloroacetic acid were purchased from Fluka (Switzerland). Ketoprofen, diclofenac sodium salt, acetonitrile, $\mathrm{D}_{2} \mathrm{O}$, DMSO-d6 and DOWEX 50WX4-50 ion-exchange resin were purchased from Aldrich (England). Acyclovir was kindly provided as a gift by Recordati (Italy).

\subsection{Synthesis of carboxymethyl scleroglucan (Scl-CM)}

Scleroglucan $(1.0 \mathrm{~g})$ was dissolved in water $(20 \mathrm{~mL})$ at $90{ }^{\circ} \mathrm{C}$ and to the solution, transferred in ice bath, $\mathrm{NaOH}(14.3 \mathrm{~g})$ was added. After complete dissolution, a solution of chloroacetic acid (5.0 g) in water $(25 \mathrm{~mL})$ was added dropwise. The mixture was maintained under stirring at $60{ }^{\circ} \mathrm{C}$ for $24 \mathrm{~h}$. After neutralization with glacial acetic acid, the solution was submitted to exhaustive dialysis. In order to obtain all the carboxylic residues in the undissociated form, the dialyzed solution was eluted through a DOWEX 50WX4-50 ion-exchange resin column previously treated with $2.0 \mathrm{M} \mathrm{HCl}$. After freezedrying, the new polymer was characterized by FT-IR and ${ }^{1} \mathrm{H}-\mathrm{NMR}$ spectra. FT-IR spectra were recorded with a Perkin Elmer Paragon 1000 spectrophotometer (USA) in the range 4,000-400 $\mathrm{cm}^{-1}$ using $\mathrm{KBr}$ pellets (number of scans 100, resolution of $1 \mathrm{~cm}^{-1}$ ). ${ }^{1} \mathrm{H}-\mathrm{NMR}$ spectra were obtained with a Bruker AC-400 instrument (Bruker, Germany) following a procedure already reported in the literature for other $(1 \rightarrow 3)$ - $\beta$-glucans [18]. In order to determine the number of carboxylic groups introduced on the scleroglucan, samples of the derivative $(100 \mathrm{mg})$ were dissolved in water and submitted to 
potentiometric titration with $1 \times 10^{-2} \mathrm{M} \mathrm{NaOH}$. The amount of acid in mmol (equal to the mmol of employed base) was inserted into the equation:

$$
\text { amount of polymer }(\mathrm{mg})=X \mathrm{PM}_{\text {repetitive unit }}+(\mathrm{mmol} \text { of acid }) \mathrm{PM}_{\text {acid group }}
$$

and allowed the calculation of $\mathrm{X}$, the mmol of repetitive units of the polymer. The ratio between the mmol of acid and the mmol of the repetitive units of the polymer multiplied by 100 gave the degree of derivatization (DD, number of carboxylic groups for 100 repetitive units) that was $65 \pm 5$.

\subsection{Studies of interaction with mucin}

Since Scl and Scl-CM do not produce clear solutions in distilled water, the polymers were centrifuged ( $15 \mathrm{~min}, 8,000 \mathrm{rpm}, 20^{\circ} \mathrm{C}$ ) in order to remove macro-aggregates eventually present in the solutions. Then the supernatant was recovered, lyophilized and the solid used for the evaluation of mucoadhesiveness. Ten $\mathrm{mg}$ of mucin were dispersed in a $0.1 \mathrm{M}$ phosphate buffered solution $(8 \mathrm{~mL}$, $\mathrm{Na}_{2} \mathrm{HPO}_{4}, \mathrm{KH}_{2} \mathrm{PO}_{4}, \mathrm{pH} 6.8$ ) at $37{ }^{\circ} \mathrm{C}$ for $24 \mathrm{~h}$ under stirring. Then Scl, Scl-CM or hyaluronic acid (HA, chosen as a positive control) $(10 \mathrm{mg})$ in $\mathrm{pH} 6.8$ phosphate buffer $(2 \mathrm{~mL})$ were added to the mucin suspension (mucin/polymer weight ratio equal to 1 ) and each sample was incubated at $37{ }^{\circ} \mathrm{C}$ under stirring for $30 \mathrm{~min}, 2,4,6,8$ and $24 \mathrm{~h}$. After each incubation time, the transmittance of the samples was recorded at $500 \mathrm{~nm}$. Turbidity of the polymer-mucin mixtures was compared with that of a mucin dispersion containing the same mucin amount as the mixtures.

\subsection{Hydrogel preparation}

To a solution of Scl-CM $(0.100 \mathrm{~g}$ in $5 \mathrm{~mL}$ of water, $\mathrm{Cp}=2 \% \mathrm{w} / \mathrm{v})$ maintained at $60{ }^{\circ} \mathrm{C}$ under stirring, different amounts of $\mathrm{CaCl}_{2} \times 2 \mathrm{H}_{2} \mathrm{O}$ were added, in order to obtain salt concentrations (Cs) of $0.05,0.10,0.25$ and $0.50 \mathrm{M}$, respectively. After complete dissolution, the solutions were left to cool to room temperature. The hydrogels containing the drugs were prepared dissolving the polymer $(0.100 \mathrm{~g}$, $\mathrm{Cp}=2 \% \mathrm{w} / \mathrm{v})$ in a solution of the drug $(0.50 \% \mathrm{w} / \mathrm{v}$ for NSAIDs, $0.25 \%$ for acyclovir $)$ maintained at $60{ }^{\circ} \mathrm{C}$. After complete dissolution, $\mathrm{CaCl}_{2} \times 2 \mathrm{H}_{2} \mathrm{O}$ was added in order to obtain $\mathrm{Cs}=0.05$ and $0.10 \mathrm{M}$ respectively.

\subsection{Release studies}

Release experiments were carried out on samples $(2.5 \mathrm{~g})$ of freshly prepared hydrogels $(\mathrm{Cp}=2 \% \mathrm{w} / \mathrm{v}$ and $\mathrm{Cs}=0.05$ and $0.10 \mathrm{M})$ containing the drug $(0.50 \% \mathrm{w} / \mathrm{v})$, with the rotating basket technique at $37.0 \pm 0.1{ }^{\circ} \mathrm{C}$ and $100 \mathrm{rpm}$ according to U.S.P. XXIV. The experiments were carried out with a SOTAX AT7 Smart (Switzerland) in 0.1M phosphate buffer ( $\mathrm{PB}, \mathrm{pH}=7.4)$ as release medium $(0.5 \mathrm{~L})$. The final concentration of the drug was always lower than $10 \%$ of its solubility in water [19], so that sink conditions could be assumed. The gel was carefully inserted into the basket and the drug release was followed by means of HPLC analysis, monitoring the amount of acyclovir at $255 \mathrm{~nm}$, ibuprofen at $215 \mathrm{~nm}$, ketoprofen and diclofenac at $253 \mathrm{~nm}$. HPLC apparatus consisted of a Perkin Elmer Series 200 LC pump, equipped with a 235 Diode Array (USA). HPLC analyses were carried out using a Merck Hibar LiChrocart $(250-4,5 \mu \mathrm{m}) \mathrm{RP}-18$ column, with a flow of $1 \mathrm{~mL} / \mathrm{min}$. $\mathrm{CH}_{3} \mathrm{CN} / \mathrm{H}_{3} \mathrm{PO}_{4} 10^{-2} \mathrm{M}$ 
mixture (7:3) was used as eluant for acyclovir and ibuprofen, $\mathrm{CH}_{3} \mathrm{CN} / \mathrm{H}_{3} \mathrm{PO}_{4} 10^{-2} \mathrm{M}$ mixture (1:1) for ketoprofen and diclofenac. All the experiments were carried out in triplicate and the results are reported as mean value $\pm \mathrm{SD}$.

\subsection{Preparation of a double-layered system}

Fifty $\mathrm{mg}$ of scleroglucan were dissolved at $60{ }^{\circ} \mathrm{C}$ in $0.10 \mathrm{M} \mathrm{CaCl}_{2}(2.5 \mathrm{~mL})$ containing $0.5 \% \mathrm{w} / \mathrm{v}$ of diclofenac sodium salt. The warm mixture was rapidly poured into a small glass (diameter=19 $\mathrm{mm}$ ). Anther $50 \mathrm{mg}$ of scleroglucan were dissolved at $60{ }^{\circ} \mathrm{C}$ in $0.05 \mathrm{M} \mathrm{CaCl}_{2}(2.5 \mathrm{~m})$ containing $0.5 \%$ of diclofenac sodium salt and the warm gel was poured carefully on the first layer already cold. The release studies of diclofenac were carried out according to the method described before and using the same dissolution apparatus equipped with paddles instead of baskets, putting the glass with the doublelayer system at the bottom of the vessel filled with $0.5 \mathrm{~L}$ of PB.

\subsection{Rheological measurements}

Rheological experiments were performed with a Haake RheoStress 300 Rotational Rheometer (Germany) equipped with a Haake DC10 thermostat. Oscillatory experiments were performed at $25.0 \pm 0.2{ }^{\circ} \mathrm{C}$ in the range $0.01-10 \mathrm{~Hz}$ on the hydrogels obtained adding a solution of $\mathrm{CaCl}_{2}$ to $2 \% \mathrm{w} / \mathrm{v}$ Scl-CM solutions in order to obtain final salt concentrations $0.05,0.10,0.25$ and $0.50 \mathrm{M}$ (Cp2-Cs0.05; Cp2-Cs0.1; Cp2-Cs0.25; Cp2-Cs0.5). Enough quantity of each sample was carefully poured to completely cover the $6 \mathrm{~cm}$ cone-plate geometry (angle of $1^{\circ}$ ). For each sample the linear viscoelastic range was evaluated: a $1 \%$ maximum deformation was used. Analogous experiments were carried out on the hydrogels loaded with diclofenac sodium salt.

\subsection{In vivo studies}

Three healthy male New Zealand White rabbits were purchased from Charles River (Calco, Lecco, Italy) and acclimated to the laboratory for a week. The rabbits were individually housed and received standard diet and tap water ad libitum. Principles of laboratory animal care (EEC Directive of 1986; 86/609/EEC) guidelines were followed.

The back of the animals was clipped free of fur with an electric clipper $24 \mathrm{~h}$ before application of the sample. For the experiment, the clipped areas of skin of each rabbit were divided into four sites with the same area $(30 \times 30 \mathrm{~mm})$. Scl, Scl-CM and the hydrogel Scl-CM with $0.1 \mathrm{M} \mathrm{CaCl}_{2}$ were applied to three sites (approximately $500 \mathrm{mg} / \mathrm{site}$ ); the last site was used as a control (no material present). The treated and the control sites were covered by gauze and the back of the rabbit was wrapped with a nonocclusive bandage, thereafter the animals were returned to their cages. After $4 \mathrm{~h}$, the bandage and the test material were removed and $1 \mathrm{~h}$ later the sites were examined for skin irritation. Observation of the sites with material and control was repeated after 24,48 and $72 \mathrm{~h}$. The reaction, defined as erythema (Er) or edema (Ed), was evaluated according to the score of the skin reactions already reported [20].

The Score of Primary Irritation (SPI) was calculated for each rabbit as the difference between the sum of the scores for erythema (Er) and edema (Ed), at 24, 48 and $72 \mathrm{~h}$ divided by the number of the 
observations for the treated sites and the sum of the scores for erythema (Er) and edema (Ed), at 24, 48 and $72 \mathrm{~h}$ divided by the number of the observations for the control sites, according to the formula:

$\mathrm{SPI}=\left[\frac{\Sigma(\mathrm{Er}+\mathrm{Ed})_{24 \mathrm{~h}}+(\mathrm{Er}+\mathrm{Ed}) 4_{8 \mathrm{~h}}+(\mathrm{Er}+\mathrm{Ed})_{72 \mathrm{~h}}}{\text { No. of observations }}\right] \mathrm{T}-\left[\frac{\sum(\mathrm{Er}+\mathrm{Ed})_{24 \mathrm{~h}}+(\mathrm{Er}+\mathrm{Ed})_{48 \mathrm{~h}}+(\mathrm{Er}+\mathrm{Ed})_{72 \mathrm{~h}}}{\text { No. of observations }}\right] \mathrm{C}$

where T, treated; C, control; Er, erythema; Ed, edema. The PII was calculated as the arithmetical mean of the SPI values of the three animals. The evaluation of PII was performed according to the categories reported in the literature [20].

\section{Conclusions}

An easy to prepare and very versatile drug delivery system was obtained from an anionic derivative of scleroglucan having carboxylic moieties. This polymer is able to form hydrogels in the presence of $\mathrm{Ca}^{+2}$. The mechanical properties of the hydrogel, as well as its ability to control the release rate of an entrapped drug, critically depends on the $\mathrm{Scl}-\mathrm{CM} / \mathrm{CaCl}_{2}$ ratio. Therefore it was observed that the amount of the released drug could be easily modulated changing the salt concentration. Moreover by an opportune combination of hydrogels prepared using different salt concentrations, it was also possible to obtain a system with zero-order release kinetics. The rheological characteristics as well as the absence of skin irritation, confirm the potential of these matrices for the development of topical delivery systems.

\section{Acknowledgements}

The authors are thankful to the Enrico and Enrica Sovena Fondation for financial support of part of this work. They wish also to thank Professor Eleonora Santucci for the useful suggestions about the double-layer system.

\section{References and Notes}

1. Coviello, T.; Matricardi, P.; Marianecci, C.; Alhaique, F. Polysaccharide hydrogels for modified release formulations. J. Control. Rel. 2007, 119, 5-24.

2. Hoffman, A.S. Hydrogels for biomedical applications. Adv. Drug Del. Rev. 2002, 54, 3-12.

3. Peppas, N.A.; Bures, P.; Leobandung, W.; Ichikawa, H. Hydrogels in pharmaceutical formulations. Eur. J. Pharm. Biopharm. 2000, 50, 27-46.

4. Kamath, K.R.; Park, K. Biodegradable hydrogels in drug delivery. Adv. Drug Deliv. Rev . 1993, $11,59-84$.

5. Coviello, T.; Palleschi, A.; Grassi, M.; Matricardi, P.; Bocchinfuso, G.; Alhaique, F. Scleroglucan: a versatile polisaccaride for modified drug delivery. Molecules 2005, 10, 6-33.

6. Kitamura, S.; Hirano, T.; Takeo, K.; Fukada, H.; Takahashi, K.; Falch, B.H.; Stokke, T. Conformational transitions of schizophyllan in aqueous alcaline solution. Biopolymers 1995, 39, 407-416. 
7. Guo, B.; Elgsaeter, A.; Stokke, B.T. Scleroglucan gel volume changes in dimethylsulphoxide/water and alkaline solutions are partly caused by polymer chain conformational transitions. Carbohydr. Polym. 1999, 39, 249-255.

8. Christensen, B.E.; Aasprong, E.; Stokke, B.T. Gelation of periodate oxidised scleroglucan (scleraldehyde). Carbohydr. Polym. 2001, 46, 241-248.

9. Stokke, B.T.; Elgsaeter, A.; Smidsrod, O.; Christensen, B.E. Carboxylation of scleroglucan for controlled crosslinking by heavy metal ions. Carbohydr. Polym. 1995, 27, 5-11.

10. Coviello, T.; Grassi, M.; Rambone, G.; Alhaique, F. A crosslinked system from scleroglucan derivative: preparation and characterization. Biomaterials 2001, 22, 1899-1909.

11. Casadei, M.A.; Matricardi, P.; Fabrizi, G.; Feeney, M.; Paolicelli, P. Physical gels of a carboxymethyl derivative of scleroglucan: synthesis and characterization. Eur. J. Pharm. Biopharm. 2007, 67, 682-689.

12. Langman, J.S. Adverse effects of conventional non-steroidal anti-inflammatory drugs on the upper gastrointestinal tract. Fundam. Clin. Pharmacol. 2003, 17, 393-403.

13. de Nooy, A.E.J.; Rori, V.; Masci, G.; Dentini, M.; Crescenzi, V. Synthesis and preliminary characterization of charged derivatives and hydrogels from scleroglucan. Carbohydr. Res. 2000, 324,116-126.

14. He, P.; Davis, S.S.; Illum, L. In vitro evaluation of the mucoadhesive properties of chitosan microspheres. Int. J. Pharm. 1998, 166, 75-88.

15. Rossi, S.; Ferrari, F.; Bonferoni, M.C.; Caramella, C. Characterization of chitosan hydrochloridemucin interaction by means of viscosimetric and turbidimetric measurements. Eur. J. Pharm. Sci. 2000, 10, 251-257.

16. Feeney, M.; Casadei, M.A.; Matricardi, P. Carboxymethyl derivative of scleroglucan: a novel thermosensitive hydrogel forming polysaccharide for drug delivery applications. J. Mater. Sci. Mater. Med. 2009, 20, 1081-1087.

17. Barry, B.W. Dermatological formulations-Percutaneous Absorption; Marcel Dekker: New York, NY, USA, 1983; vol.18, pp. 351-396.

18. Ensley, H.E.; Tobias, B.; Pretus, H.A.; McNamee, R.B.; Jones, E.L.; Browder, I.W.; Williams, D.L. NMR spectral analysis of a water-insoluble $(1 \rightarrow 3)$-beta-D-glucan isolated from Saccharomyces cerevisiae. Carbohydr. Res. 1994, 258, 307-311.

19. Bergström, C.A.S.; Norinder, U.; Luthman, K.P.; Artursson, P. Experimental and computational screening models for prediction of aqueous drug solubility. Pharm. Res. 2002, 19, 182-188.

20. Draize, J.H.; Woodard, G.; Calvery, H.O. Methods for the study of irritation and toxicity of substances applied topically to the skin and the mucous membranes. J. Pharmacol. Exp. Ther. 1944, 82, 377-390.

Sample Availability: Samples of the compounds Scl-CM are available from the authors.

(C) 2009 by the authors; licensee Molecular Diversity Preservation International, Basel, Switzerland. This article is an open-access article distributed under the terms and conditions of the Creative Commons Attribution license (http://creativecommons.org/licenses/by/3.0/). 